\title{
A Comparative Analysis of the Metabolic and Coagulative Profiles in Patients with Idiopathic Scoliosis, Congenital Scoliosis and Healthy Controls: A Case-Control Study
}

\author{
Kaustubh Ahuja ${ }^{1}$, Bhavuk Garg ${ }^{1}$, Buddhadev Chowdhuri ${ }^{1}$, Raj Kumar Yadav ${ }^{2}$, Pradeep Kumar Chaturvedi ${ }^{3}$ \\ ${ }^{1}$ Department of Orthopaedics, All India Institute Of Medical Sciences, New Delhi, India \\ ${ }^{2}$ Department of Physiology, All India Institute Of Medical Sciences, New Delhi, India \\ ${ }^{3}$ Department of Reproductive Medicine, All India Institute Of Medical Sciences Sciences, New Delhi, India
}

Study Design: Single-center, observational, case-control study.

Purpose: Comparison and analysis of the metabolic and coagulative profiles in patients with idiopathic scoliosis, patients with congenital scoliosis, and healthy controls.

Overview of Literature: Serum melatonin deficiency has been a controversial topic in the etiopathogenesis of scoliosis. Low bone mineral density, low vitamin D3 levels, and high parathyroid hormone levels are common metabolic abnormalities associated with scoliosis that may be responsible for its pathogenesis. In addition to metabolic defects, several studies have shown coagulation defects that either persist from the preoperative period or occur during surgery and usually lead to more than the expected amount of blood loss in patients undergoing deformity correction for scoliosis.

Methods: The study population ( $n=73$ ) was classified into those having congenital scoliosis ( $n=31)$, those with idiopathic scoliosis $(n=30)$, and healthy controls $(n=12)$. After detailed clinicoradiological evaluation of all the subjects, 10-mL blood samples were collected, measured, and analyzed for various metabolic and coagulation parameters.

Results: The mean serum melatonin levels in patients with idiopathic scoliosis were significantly lower than those in the healthy controls. Although the mean serum melatonin level in the congenital group was also low, the difference was not statistically significant. Serum alkaline phosphatase and parathyroid hormone levels were higher in the scoliosis groups, whereas the vitamin D level was lower. No differences were observed in the coagulation profiles of the different groups.

Conclusions: Low serum melatonin levels associated with scoliosis can be a cause or an effect of scoliosis. Moreover, low bone mineral density, high bone turn over, and negative calcium balance appear to play an important role in the progression, if not the onset, of the deformity.

Keywords: Scoliosis; Spinal curvature; Melatonin; Vitamin D

Received Feb 21, 2018; Revised Mar 27, 2018; Accepted May 2, 2018

Corresponding author: Bhavuk Garg

Department of Orthopaedics, All India Institute of Medical Sciences, Ansari nagar, New Delhi -110029, India

Tel: +91-9899558021, Fax: +91-11-26588663, E-mail: drbhavukgarg@gmail.com 


\section{Introduction}

Scoliosis is the most commonly diagnosed spinal deformity that affects $2 \%-4 \%$ of all children [1]. It is a threedimensional deformity, with extension of the intervertebral segment in the sagittal plane inducing lordosis, lateral intervertebral tilting in the frontal plane, and rotation in the axial planes of the spine. Any lateral deviation of the spine of $>10^{\circ}$ on an anterior-posterior radiograph of the whole spine associated with vertebral rotation is considered to indicate scoliosis [2]. Despite the availability of several studies on the subject, the etiology and pathogenesis remain unclear. Apart from genetics [3,4], asymmetric growth and development of the vertebrae, mechanics of an upright spine [5], and laxity of the paraspinal ligaments and muscles [6], there has been considerable interest in the metabolic profile of patients with idiopathic scoliosis. Melatonin deficiency is believed to play an important role in the development and progression of spinal deformity in adolescent scoliosis $[7,8]$, although this hypothesis is controversial $[9,10]$. Low bone mineral density (BMD), low vitamin D3 level, and high parathyroid hormone (PTH) level are among other metabolic abnormalities that are commonly associated with scoliosis; these may or may not play a role in its pathogenesis $[1,11]$.

In addition to metabolic defects, several studies have shown coagulation defects that either persist from the preoperative period or occur during surgery and usually lead to more than the expected amount of blood loss in patients undergoing deformity correction for scoliosis [12-14]. In the present study, we analyzed the metabolic and coagulative profiles of patients with idiopathic and congenital scoliosis and compared them with each other and with those of healthy controls. Clinical identification of the metabolic factors responsible for scoliosis may be vital for the formulation of pre-symptomatic diagnostic tests that could help identify asymptomatic children who are at risk of developing scoliosis and symptomatic children at risk of disease progression. It may also help identify targeted pharmacotherapy to stop disease progression in children with scoliosis. Preoperative identification of specific coagulation defects may enable the use of targeted, specific interventions to reduce the risk of bleeding.

\section{Materials and Methods}

An observational case-control study was performed to compare the metabolic parameters and coagulation profiles of patients with idiopathic and congenital scoliosis as well as healthy controls. Ethical clearance was obtained from the institutional ethics committee of the All India Institute of Medical Sciences (IRB approval no., IESC/ T-589/2015).

A total of 73 consecutive patients who presented at the orthopedic outpatient department of the scoliosis clinic in our hospital from August 1, 2015, to December 31, 2016, were enrolled after they provided informed consent. The study population was divided into three groups, as those with congenital scoliosis (group $A, n=31$ ), those with idiopathic scoliosis (group $\mathrm{B}, \mathrm{n}=30$ ), and healthy controls (group $\mathrm{C}, \mathrm{n}=12$ ). The inclusion criteria for the cases were as follows: (1) patient age $<18$ years, (2) idiopathic or congenital scoliosis, and (3) no history of any previous surgery. The inclusion criteria for the controls were as follows: (1) age <18 years, (2) old fractures healed with conservative management, (3) soft tissue injuries of the ankle and the elbow, and (4) low back pain. The exclusion criteria for the cases were as follows: (1) patient age $>18$ years; (2) previous surgery; (3) scoliotic deformity as a previously diagnosed part of a syndrome; and (4) neuromuscular, mesenchymal, or adult scoliosis. Exclusion criteria for the controls were as follows: (1) patient age $>18$ years; (2) any neoplastic condition; (3) renal or hepatic disorders; (4) personal or family history of scoliosis or any neurologic, endocrine, or psychiatric disorders; and (5) clinical evidence of any spinal deformity.

\section{Clinical assessment}

All the patients were subjected to detailed standard clinicoradiological examination for scoliosis, including general examination for the presence of comorbidities, presence of other physical malformations and congenital deformities, local examination of the spine, rib hump, flexibility, and Cobb's angle. Antero posterior, lateral, and side-bending full-length radiographs were obtained for all subjects (Fig. 1). Patients who met the inclusion criteria were enrolled.

\section{Sample collection}

Samples were collected from all the subjects after overnight fasting. It was ensured that the lights were switched off during the bed time hours on the previous night. Blood samples $(10 \mathrm{~mL})$ were collected by the first author 

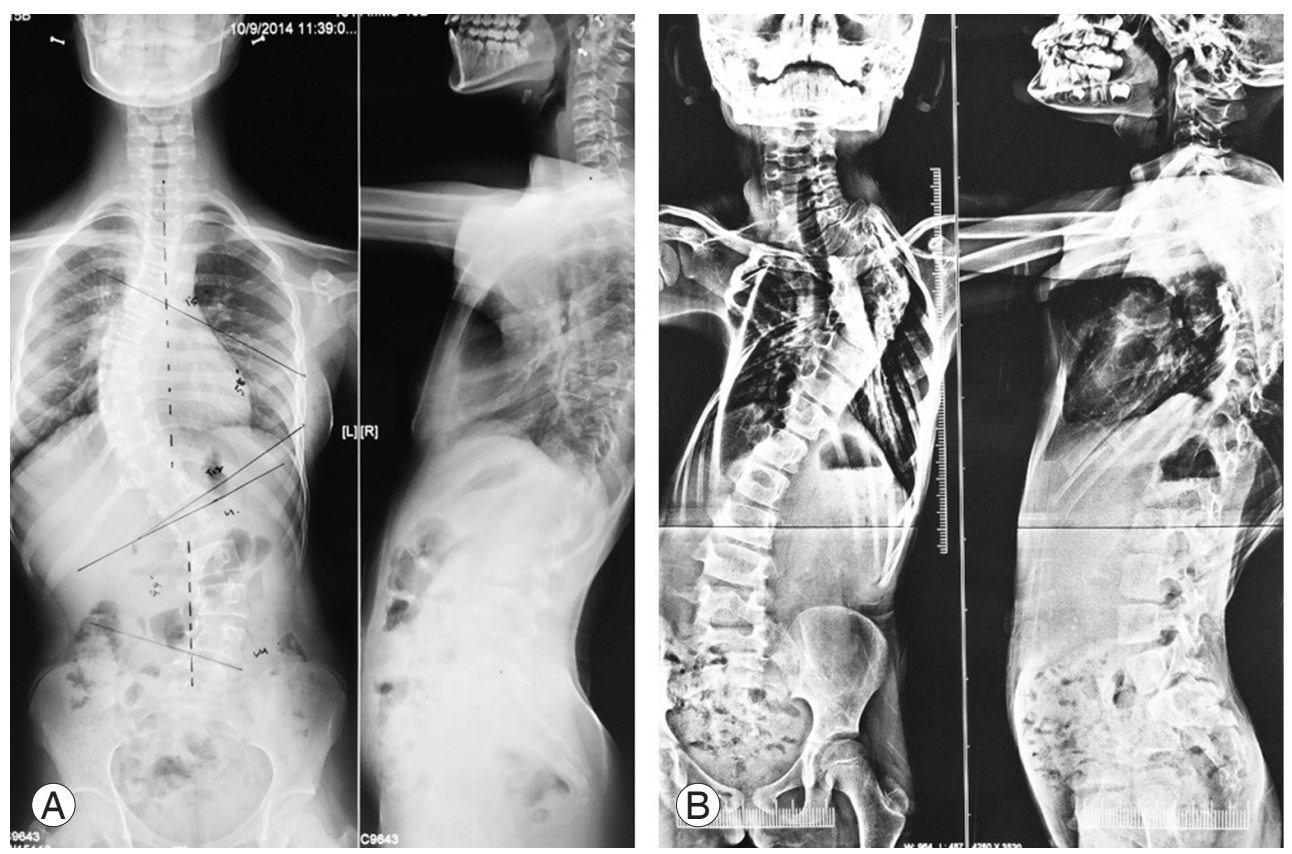

Fig. 1. (A, B) AP and lateral radiographs of a 13-year-old girl with idiopathic thoraco-lumbar scoliosis. (C, D) AP and lateral radiographs of a 7-year-old girl with congenital thoracic scoliosis showing hemivertebrae at D3, 4, 5, and 6 levels with a bar between D5 and 6. AP, anterior-posterior.

(K. A.) under aseptic conditions from the median cubital and cephalic veins from the non-dominant arm after tourniquet application. All the samples were collected between 12 noon and 1:00 PM. After centrifugation, the serum was separated and stored at $-40^{\circ}$ Celsius for analysis. The following parameters were evaluated for all the subjects.

Metabolic parameters were as follows: serum melatonin (pg/mL, ELISA MT; Cloud-Clone Corp., Wuhan, China); serum calcium ( $\mathrm{mg} / \mathrm{dL}$, Modular P electrolyte analyzer, ISE 900; Roche Diagnostic Corp., Indianapolis, IN, USA); serum alkaline phosphatase (ALP [IU/L], Modular P, ISE 900; Roche Diagnostic Corp.); serum phosphate (mg/dL, Modular P electrolyte analyzer, ISE 900; Roche Diagnostic Corp.); serum PTH (pg/mL, Architect Intact PTH; Abbott Laboratories, Wiesbaden, Germany); and serum 1,25-OH vitamin D (ng/mL, Architect VIT D; Abbott Laboratories). Coagulation profile was as follows: prothrombin time (PT, CA 1500; Sysmex America Inc., Mundlein, IL, USA); international normalized ratio (INR, CA 1500; Sysmex America Inc.); and activated partial thromboplastin time (APTT, CA 1500; Sysmex America Inc.).

\section{Statistical analyses}

Data were plotted in Microsoft Excel sheet (Microsoft
Corp., Redmond, WA, USA) and analyzed using the statistical software STATA ver. 14.0 (Stata Corp., College Station, TX, USA). Categorical data are expressed as frequencies and percentages. Normal and non-normal qualitative data are expressed as mean \pm standard deviation and median (minimum and maximum) values, respectively. One way analysis of variance followed by Bonferroni multiple comparison test was used to compare the continuous normal variables among the groups, while the KruskalWallis $\mathrm{H}$ test followed by Dunn test was used to compare the continuous variables following non-normal distribution. In addition, Pearson and Spearman correlation coefficients were used to determine the correlation among variables and the strength of such correlations. All $p$-value $<0.05$ was considered statistically significant.

\section{Results}

A total of 73 subjects, including 31 patients in group A (congenital scoliosis), 30 in group B (idiopathic scoliosis), and 12 in group $\mathrm{C}$ (healthy controls) were enrolled. All these patients were evaluated during their second visit to our outpatient department for various metabolic and coagulation parameters; their values were compared among the three groups. 


\section{Demographic and baseline characteristics}

1) Age

All the subjects were 2-18 years of age. Group A had a mean age of $13.5 \pm 3.3$ years with $12.9 \pm 5.2$ for boys and $13.9 \pm 3.0$ for girls; the minimum age was 4 years, and the maximum age was 17 years. Group B had a mean age of $13.4 \pm 3.0$ years, with $13.6 \pm 3.5$ for boys and $13.2 \pm 3.2$ for girls; the minimum age was 5 years, and maximum age was 18 years. Group C had a mean age of $12.9 \pm 4.4$ years, with $12.5 \pm 3.2$ for boys and 13.2 \pm 3.2 for girls; the minimum age was 5 years, and the maximum age was 18 years. There was no statistically significant difference in the age of the three groups $(p=0.86)$.

\section{2) Sex}

Of the 31 patients in group A, 15 (48.3\%) were girls and $16(51.7 \%)$ were boys. In group B, of the 30 patients, 15 (50\%) were girls and 15 were boys (45\%). In group C, six (50\%) of the 12 subjects were girls, and six (50\%) were boys.

\section{3) Cobb's angle}

The Cobb's angles of all the subjects were measured by a single investigator (K. A.). The mean value of the Cobb's angle for group A was $44.7^{\circ} \pm 20.2^{\circ}$, while that for group B was $49.4^{\circ} \pm 18.0^{\circ}$. There was no statistically significant difference between the Cobb's angles of the two groups.

\section{Metabolic parameters}

\section{1) Serum melatonin}

The mean value of the serum melatonin in group A was $142.27 \pm 34.6 \mathrm{pg} / \mathrm{mL}$, lower than that in the controls (group C) who had a mean melatonin value of $184.2 \pm 64.8 \mathrm{pg} / \mathrm{mL}$; however, this difference was not statistically significant $(p=0.11)$ (Fig. 2). The mean melatonin value for group B was $127.19 \pm 75.1 \mathrm{pg} / \mathrm{mL}$. This value was much lower than that for the controls, and the difference was statistically significant $(p=0.02)$. The boys in group B had a mean serum melatonin level of $107.7 \pm 8.4 \mathrm{pg} / \mathrm{mL}$, while the girls in group B had a mean value of $140.1 \pm 24.1 \mathrm{pg} / \mathrm{mL}(p=0.3)$; the mean serum melatonin level of the control group boys was $155.4 \pm 13.3 \mathrm{pg} / \mathrm{mL}$, while that of the control group girls was $241.9 \pm 37.0 \mathrm{pg} / \mathrm{ml}$. The difference between both the sexes was statistically significant $(p<0.0001)$. On applying the correlation statistics, the Cobb's angle was in-

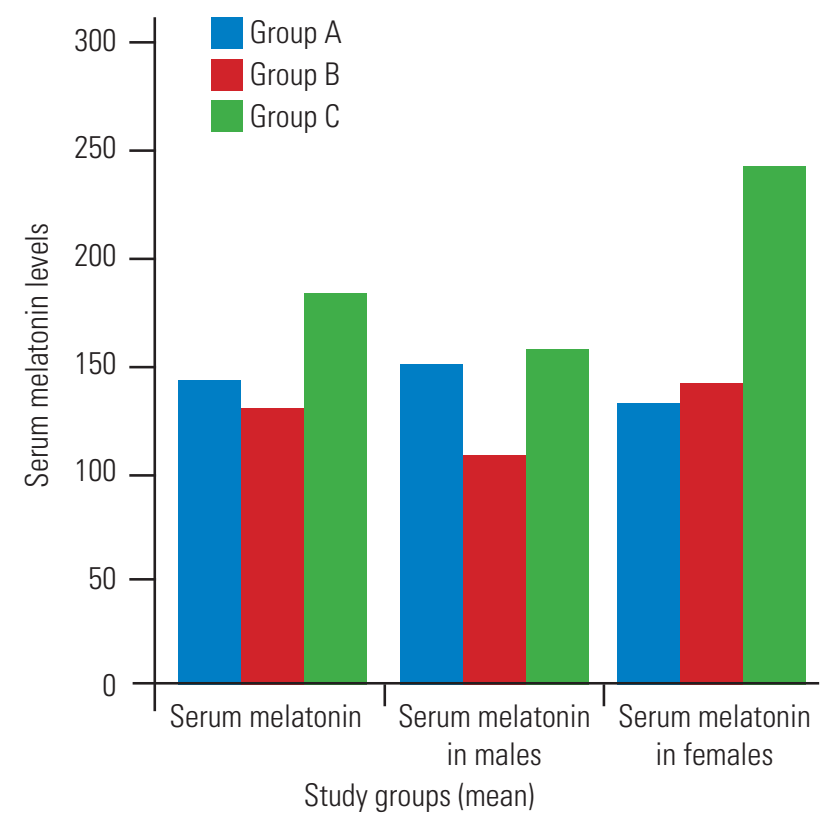

Fig. 2. Serum melatonin levels in all three study groups as well as sex-based classification of the values.

versely related to the serum melatonin levels in group B, and the correlation was statistically significant $(p=0.02)$. There was no correlation between the serum melatonin levels and the Cobb's angle in group A.

\section{2) Serum calcium}

The mean serum calcium level was $9.3 \pm 0.49 \mathrm{mg} / \mathrm{dL}$ in group $\mathrm{A}, 9.2 \pm 0.5 \mathrm{mg} / \mathrm{dL}$ in group $\mathrm{B}$, and $9.5 \pm 0.3 \mathrm{mg} / \mathrm{dL}$ in group C. None of the values was significantly different from the others. There was no difference in the serum calcium levels of the boys and girls across all the groups.

3) Serum phosphate

Mean serum phosphate levels of group A $(4.9 \pm 0.82 \mathrm{mg} /$ $\mathrm{dL})$ and group $\mathrm{B}(4.8 \pm 0.4 \mathrm{mg} / \mathrm{dL})$ were significantly higher than those of group C $(3.9 \pm 0.34 \mathrm{mg} / \mathrm{dL})(p<0.001)$.

4) Serum alkaline phosphatase

The mean serum ALP level of group A was 501.2 \pm 183.2 IU/L, that of group B was $486.5 \pm 266.1 \mathrm{IU} / \mathrm{L}$, and that of group C was 323.4 \pm 135.2 IU/L. Serum ALP levels in groups $\mathrm{A}$ and $\mathrm{B}$ were significantly higher than that in group $\mathrm{C}(p<0.001$ and $p<0.04$, respectively). The ALP values of girls $(509.2 \pm 45.7 \mathrm{IU} / \mathrm{L})$ were much higher than those of boys $(421.8 \pm 26.9 \mathrm{IU} / \mathrm{L})$ within as well as across the groups. 


\section{5) Serum $1,25-\mathrm{OH}$ vitamin $\mathrm{D}$}

The mean serum 1,25-OH vitamin D levels of group A, group $\mathrm{B}$, and group $\mathrm{C}$ were $15.3 \pm 10.9 \mathrm{ng} / \mathrm{mL}, 13.4 \pm 11.1$ $\mathrm{ng} / \mathrm{mL}$, and $28.2 \pm 20.8 \mathrm{ng} / \mathrm{mL}$, respectively. The vitamin $\mathrm{D}$ levels in groups A and B were significantly lower than that in group C ( $p=0.01$ and $p<0.001$, respectively).

6) Serum parathyroid hormone levels

The mean serum PTH levels of group A, group B, and group $\mathrm{C}$ were $49.6 \pm 27.6 \mathrm{pg} / \mathrm{mL}, 50.5 \pm 24.6 \mathrm{pg} / \mathrm{mL}$, and $32.0 \pm 26.0 \mathrm{pg} / \mathrm{mL}$, respectively. The serum PTH levels in groups A and B were significantly higher than that in group $C$ ( $p=0.01$ and $p=0.00$, respectively).

\section{Coagulation studies}

\section{1) Prothrombin time}

The mean PT in group A was $13.0 \pm 1.7$ seconds; this was significantly higher than that in group B (PT 12.2 \pm 0.75 seconds, $p<0.05)$ although both were within the normal range. The PT in group A was also higher than that in group C (PT 12.4 \pm 0.74 seconds); however, the difference was not statistically significant.

2) Activated partial thromboplastin time

The mean APTT levels of group A, group B, and group C (35.6 $\pm 6.6,32.2 \pm 3.6$, and $38.3 \pm 7.4$ seconds, respectively) were within the normal range. We did not find any preoperative coagulation abnormality in any patient. All the results are summarized in Table 1.

\section{Discussion}

The pathogenesis and mechanisms leading to threedimensional deformity in a scoliotic spine have been a constant topic of discussion among scientists worldwide. Several factors are speculated to play a role in the pathogenesis of scoliosis. In this study, we analyzed several metabolic factors implicated in the causation of scoliosis. In addition, we studied the coagulative profiles in scoliosis patients.

Table 1. Summary of various demographic parameters, metabolic and coagulative parameters in the three test groups and their statistical comparison with respective $p$-values

\begin{tabular}{|c|c|c|c|c|c|c|}
\hline \multirow{2}{*}{ Variable } & \multirow{2}{*}{$\mathrm{CON}$} & \multirow{2}{*}{$\mathrm{AD}$} & \multirow{2}{*}{ CTRL } & \multicolumn{3}{|c|}{$\begin{array}{l}\text { Multiple comparison } \\
\text { (p-value) }\end{array}$} \\
\hline & & & & $\begin{array}{l}\text { CON vs. } \\
\text { CTRL }\end{array}$ & $\begin{array}{l}\text { AD vs. } \\
\text { CTRL }\end{array}$ & $\begin{array}{l}\text { AD vs. } \\
\text { CON }\end{array}$ \\
\hline Age (yr) & $13.5 \pm 3.3$ & $13.4 \pm 3.0$ & $12.9 \pm 4.4$ & & & \\
\hline \multicolumn{7}{|l|}{ Sex } \\
\hline Male & $16(53.3)$ & $9(36.0)$ & $8(66.6)$ & & & \\
\hline Female & $14(46.6)$ & $15(60.0)$ & $3(25.0)$ & & & \\
\hline Cobbs angle $\left(^{\circ}\right)$ & $44.7 \pm 20.2$ & $49.4 \pm 18.07$ & Not applicable & & & \\
\hline Serum melatonin (pg/mL) & $142.27 \pm 34.6$ & $127.19 \pm 75.1$ & $184.2 \pm 64.8$ & 0.11 & 0.02 & 0.99 \\
\hline Serum calcium (mg/dL) & $9.34 \pm 0.49$ & $9.2 \pm 0.54$ & $9.50 \pm 0.31$ & & & \\
\hline Serum phosphate (mg/dL) & $4.9 \pm 0.82$ & $4.0 \pm 0.40$ & $3.9 \pm 0.34$ & $<0.01$ & 0.99 & $<0.01$ \\
\hline International normalized ratio & $1.17 \pm 016$ & $1.3 \pm 0.07$ & $1.19 \pm 0.06$ & & & \\
\hline Prothrombin time (sec) & $13.09 \pm 1.70$ & $12.2 \pm 0.75$ & $12.4 \pm 0.74$ & 0.39 & 0.99 & 0.06 \\
\hline $\begin{array}{l}\text { Activated partial thromboplastin } \\
\text { time }(\mathrm{sec})\end{array}$ & $35.6 \pm 6.6$ & $32.2 \pm 3.6$ & $38.3 \pm 2.4$ & 0.52 & 0.01 & 0.11 \\
\hline Alkaline phosphatase (IU/L) & $501.2 \pm 183.2(162-881)$ & $486.5 \pm 266.1(174-1,502)$ & $323.4 \pm 135.2(26-475)$ & $<0.01$ & 0.04 & 0.76 \\
\hline Vitamin D (ng/mL) & $15.3 \pm 10.9(3.2-58.5)$ & $13.4 \pm 7.0(5.1-32.2)$ & $28.2 \pm 20.8(8.4-79.7)$ & 0.01 & $<0.01$ & 0.99 \\
\hline Parathyroid hormone (pg/mL) & $49.6 \pm 27.6(14.7-122.4)$ & $50.5 \pm 24.6(10.1-113.2)$ & $32.0 \pm 26.0(3.6-109.1)$ & 0.01 & $<0.01$ & 0.91 \\
\hline
\end{tabular}

Values are presented as mean \pm SD, number (\%), or mean \pm SD (min-max). Bold type is considered statistically significant. CON, congenital; $A D$, adolescent; CTRL, control; SD, standard deviation. 


\section{Serum melatonin}

Melatonin is a pineal hormone biochemically derived from the essential amino acid tryptophan $[15,16]$. The hypothesis that melatonin plays a role in the pathogenesis of scoliosis originated from experiments conducted by Thillard [17] in 1959 wherein he was able to induce scoliosis-like deformity in chicken by resecting their pineal glands. Thereafter, several experiments have been performed by Machida et al. $[18,19]$ that indicate the role of melatonin deficiency in producing scoliotic deformity in chicken and rats $[18,19]$. In another study, Machida et al. [8] reported an association between low melatonin levels and poor prognosis of adolescent scoliosis; they also reported that melatonin supplementation helped in stopping the progress of the curve, especially when the curve was less than $35^{\circ}$. In several human experiments, scientists have found contradicting results that have raised doubts about previous claims stating serum melatonin deficiency as the major factor in the development of scoliosis. Fagan et al. [10] found no significant differences between the urinary melatonin excretion of patients with adolescent idiopathic scoliosis and controls in a fracture clinic. Bagnall et al. [9] compared afternoon and midnight serum melatonin levels in seven girls with adolescent idiopathic scoliosis and seven controls to find no significant difference between the values.

In our study, the serum melatonin levels of patients with idiopathic scoliosis were significantly lower than those in controls. These findings contradict the report by Bagnall et al. [9]. One probable reason that could explain this could be the difference in the disease stage of the patients because Bagnall et al. [9] only included scoliotic deformities that had not progressed during the previous 2 years. This may indicate that melatonin deficiency is an indicator of disease progression, not the disease itself. In this study, the serum melatonin levels were found to be inversely correlated with the Cobb's angle in patients with idiopathic scoliosis. This indirectly points toward melatonin deficiency as an indicator of disease progression.

Serum melatonin levels in patients with congenital scoliosis are lower than those in controls. Low serum melatonin levels in congenital scoliosis, and in idiopathic scoliosis, can probably be attributable to the fact that body posture affects melatonin secretion in humans [20]. Thus, melatonin deficiency in patients with idiopathic and congenital scoliosis may be an effect rather than a cause of scoliosis. This hypothesis can well explain the inverse relationship between Cobb's angle and melatonin deficiency in idiopathic scoliosis patients.

\section{Serum calcium, phosphorus, alkaline phosphatase, vitamin $\mathrm{D} 3$, and parathyroid hormone}

Several studies have assessed the serum vitamin D3 levels in patients with scoliosis and compared them with those in healthy individuals. All studies report moderate to severe deficiency of vitamin D3 in patients with scoliosis than in controls $[1,11]$. Our study revealed similar results, with the mean levels of serum vitamin D3 in patients with congenital and adolescent scoliosis being significantly lower than in controls. Across groups, the serum vitamin D3 levels were significantly higher in boys than in girls. Within the groups, although levels were higher in boys, the difference was not statistically significant.

Vitamin D deficiency leads to negative calcium balance that results in increased serum PTH levels. These high PTH levels increase the bone turn over and bone demineralization. Although serum calcium levels in these patients are well maintained because of these compensatory mechanisms, BMD is severely affected, as shown in many studies comparing the BMDs of patients with idiopathic scoliosis and controls. In our study, BMDs were not analyzed owing to logistical reasons. Serum PTH levels in patients with idiopathic scoliosis and congenital scoliosis were significantly higher than that in controls, indicating toward serum vitamin D3 insufficiency in these individuals that, due to the loss of negative feedback, could have led to these higher PTH levels. As expected, the serum calcium levels were within normal limits in all three groups with no significant difference. Serum ALP levels of the patients was significantly higher than that of the controls; this may be indicative of high bone turn observed in these patients. High bone turn over along with low BMD in scoliosis patients presents a bone model that closely simulates the model created in post-menopausal osteoporosis, wherein the bone-specific and total ALP levels increase with age. Although the precise reason for this rise is highly debatable, several theories have been put proposed. Kamimura et al. [21] speculated that the cause of the increase in bone turnover with aging and back pain may be undiagnosed vertebral fractures, and it could be a response to fracture healing. In a study by Mukaiyama et al. [22], bisphosphonate therapy administered to post- 
menopausal women lowered the total ALP levels, and this decrease strongly correlated with decrease in bone-specific ALP. Decrease in the estrogen level of post-menopausal women enhances osteoclast activation due to decreased expression of osteoprotegerin protein, an inhibitor of receptor activator of nuclear factor kappa-B ligand from the osteoblasts and stromal cells. It is well known that the activity of osteoblasts and osteoclasts is coupled by osteoclast derived complement component 3a [23]. This increased activity of osteoblasts leads to an elevated ALP levels. Bisphosphonates have not yet been used in scoliosis patients; however, their use may stop or even reverse scoliosis progression.

\section{Coagulation profile}

Patients undergoing posterior instrumentation for scoliosis or other spinal deformities have an increased risk of excessive intraoperative bleeding. The exact cause of this excessive bleeding remains unclear; however, few theories have been proposed to explain the failed hemostasis, including dilutional coagulopathy that reduces the concentration of the coagulation factors and platelets [24,25], consumption coagulopathy following the activation of the fibrinolytic pathway, abnormal levels of calmodulin that affects platelet function, or inherent coagulation abnormalities in scoliosis patients [26].

In this study, we measured and compared PT, APTT, and INR values of the patients with adolescent scoliosis, those with congenital scoliosis, and controls. Ryan et al. [12] reported that $62 \%$ of scoliosis patients have an abnormal PT compared to $33 \%$ of controls. Moreover, factor X levels were significantly lower in patients than in healthy controls [12]. Several studies have focused on the derangement of coagulation parameters during the surgery, rather than on preoperative abnormalities for the higher blood loss. Stanitski et al. [27] attempted to predict the bleeding tendencies among 36 patients and found an increase in the PT. Bosch et al. [13] used thromboelastography and serial PT, APTT, and fibrinolysis score to concluded that the mechanism for increased bleeding in patients with idiopathic scoliosis undergoing posterior spinal fusion appeared to be fibrinolysis; they recommended the routine use of antifibrinolytics in scoliosis surgery.

In this study, we found no significant difference in the PT of the adolescent scoliosis patients and controls; however, there was a significant difference between patients with congenital scoliosis and adolescent scoliosis; patients with congenital scoliosis had higher PT values than those with adolescent scoliosis, although both values were within the normal range. APTT values were within the normal range for all three groups. Considerable importance has been given to the bleeding tendencies of patients during posterior instrumentation observed in adolescent scoliosis. In the present study, we attempted to evaluate whether any preoperative coagulation disturbance existed in patients with adolescent or congenital scoliosis. Our results completely contradicted the findings of Ryan et al. [12] who found coagulation defects in $62 \%$ of the patients with scoliotic deformities. However, our findings support the report of Bosch et al. [13] and Brenn et al. [14] who found that the preoperative coagulation profiles of scoliosis patients were normal. Both studies suspected the changes in the hemostatic mechanisms in the intraoperative period to be responsible for the high blood loss during surgery for scoliosis $[13,14]$.

The relatively small sample size is a limitation of our study. However, to our knowledge, this is the first study to include most of the metabolic parameters associated with the etiopathogenesis of scoliosis along with coagulative screening. In addition, unlike most previous studies that included only adolescent girls, we included both boys and girls aged $<18$ years with idiopathic scoliosis. To the best of our knowledge, no study has included congenital scoliosis patients in their sample.

Treatment strategies for scoliosis that have been used thus far have been curative in nature. The primary reason for the inability to develop a preventive strategy is the lack of understanding of the etiopathogenesis of scoliosis. A detailed understanding of these is crucial for developing targeted pharmacotherapy and other treatment strategies to prevent the onset and progression of scoliosis.

\section{Conclusions}

Through this study, we conclude that low serum melatonin levels associated with scoliosis can be a cause or an effect of scoliosis, although further research is required to confirm this hypothesis. Moreover, low bone mineral density, high bone turn over, and negative calcium balance appear to play an important role in the progression, if not the onset, of the deformity. 


\section{Conflict of Interest}

No potential conflict of interest relevant to this article was reported.

\section{Acknowledgments}

Funding of 650 US dollar for diagnostic kits was obtained from Orthopedic Research Society of All India Institute of Medical Sciences. There are no study-specific conflicts of interest-associated biases to be disclosed by any of the authors.

\section{Author Contributions}

Kaustubh Ahuja: the conception and design, data acquisition, analysis of data, and drafting of the manuscript; Bhavuk Garg: critical revision, obtaining funding, and supervision; Buddhadev Chowdhury: critical revision, administrative support, and supervision; Raj Kumar Yadav: data acquisition, analysis of data, administrative support, and supervision; and Pradeep Kumar Chaturved: data acquisition, analysis of data, administrative support, and supervision.

\section{References}

1. Batista R, Martins DE, Hayashi LF, Lazaretti-Castro M, Puertas EB, Wajchenberg M. Association between vitamin D serum levels and adolescent idiopathic scoliosis. Scoliosis 2014;9:O45.

2. Herring JA. Tachdjian's pediatric orthopaedics: from the Texas Scottish Rite Hospital for Children. 5th ed. Philadelphia (PA): Elsevier; 2014.

3. Wynne-Davies R. Familial (idiopathic) scoliosis: a family survey. J Bone Joint Surg Br 1968;50:24-30.

4. Robin GC, Cohen T. Familial scoliosis: a clinical report. J Bone Joint Surg Br 1975;57:146-8.

5. Machida M, Murai I, Miyashita Y, Dubousset J, Yamada T, Kimura J. Pathogenesis of idiopathic scoliosis: experimental study in rats. Spine (Phila Pa 1976) 1999;24:1985-9.

6. Michelsson JE. The development of spinal deformity in experimental scoliosis. Acta Orthop Scand Suppl 1965:Suppl 81:1-91.

7. Machida M, Dubousset J, Imamura Y, Iwaya T, Yamada T, Kimura J. Role of melatonin deficiency in the development of scoliosis in pinealectomised chickens. J Bone Joint Surg Br 1995;77:134-8.

8. Machida M, Dubousset J, Yamada T, Kimura J. Serum melatonin levels in adolescent idiopathic scoliosis prediction and prevention for curve progression: a prospective study. J Pineal Res 2009;46:344-8.

9. Bagnall KM, Raso VJ, Hill DL, et al. Melatonin levels in idiopathic scoliosis: diurnal and nocturnal serum melatonin levels in girls with adolescent idiopathic scoliosis. Spine (Phila Pa 1976) 1996;21:1974-8.

10. Fagan AB, Kennaway DJ, Sutherland AD. Total 24hour melatonin secretion in adolescent idiopathic scoliosis: a case-control study. Spine (Phila Pa 1976) 1998;23:41-6.

11. Balioglu MB, Aydin C, Kargin D, et al. Vitamin-D measurement in patients with adolescent idiopathic scoliosis. J Pediatr Orthop B 2017;26:48-52.

12. Ryan KM, O'Brien K, Regan I, et al. The prevalence of abnormal preoperative coagulation tests in pediatric patients undergoing spinal surgery for scoliosis. Spine J 2015;15:1217-22.

13. Bosch P, Kenkre TS, Londino JA, Cassara A, Yang C, Waters JH. Coagulation profile of patients with adolescent idiopathic scoliosis undergoing posterior spinal fusion. J Bone Joint Surg Am 2016;98:e88.

14. Brenn BR, Theroux MC, Dabney KW, Miller F. Clotting parameters and thromboelastography in children with neuromuscular and idiopathic scoliosis undergoing posterior spinal fusion. Spine (Phila $\mathrm{Pa}$ 1976) 2004;29:E310-4.

15. Neuwelt EA, Mickey B, Lewy AJ. The importance of melatonin and tumor markers in pineal tumors. J Neural Transm Suppl 1986;21:397-413.

16. Minneman KP, Wurtman RJ. The pharmacology of the pineal gland. Annu Rev Pharmacol Toxicol 1976;16:33-51.

17. Thillard MJ. Vertebral column deformities following epiphysectomy in the chick. C R Hebd Seances Acad Sci 1959;248:1238-40.

18. Machida M, Dubousset J, Imamura Y, Miyashita Y, Yamada T, Kimura J. Melatonin: a possible role in pathogenesis of adolescent idiopathic scoliosis. Spine (Phila Pa 1976) 1996;21:1147-52.

19. Machida M, Saito M, Dubousset J, Yamada T, Kimura J, Shibasaki K. Pathological mechanism of idiopathic scoliosis: experimental scoliosis in pinealectomized rats. Eur Spine J 2005;14:843-8. 
20. Deacon S, Arendt J. Posture influences melatonin concentrations in plasma and saliva in humans. Neurosci Lett 1994;167:191-4.

21. Kamimura M, Uchiyama S, Takahara K, Hashidate H, Kawaguchi A, Nakagawa H. Urinary excretion of type I collagen cross-linked N-telopeptide and serum bone-specific alkaline phosphatase analysis to determine the correlation of age and back-pain related changes in elderly women. J Bone Miner Metab 2005;23:495-500.

22. Mukaiyama K, Kamimura M, Uchiyama S, Ikegami S, Nakamura Y, Kato H. Elevation of serum alkaline phosphatase (ALP) level in postmenopausal women is caused by high bone turnover. Aging Clin Exp Res 2015;27:413-8.

23. Matsuoka K, Park KA, Ito M, Ikeda K, Takeshita S. Osteoclast-derived complement component 3a stimulates osteoblast differentiation. J Bone Miner Res 2014;29:1522-30.
24. Murray DJ, Pennell BJ, Weinstein SL, Olson JD. Packed red cells in acute blood loss: dilutional coagulopathy as a cause of surgical bleeding. Anesth Analg 1995;80:336-42.

25. Horlocker TT, Nuttall GA, Dekutoski MB, Bryant SC. The accuracy of coagulation tests during spinal fusion and instrumentation. Anesth Analg 2001;93:338.

26. Ho WK, Baccala M, Thom J, Eikelboom JW. High prevalence of abnormal preoperative coagulation tests in patients with adolescent idiopathic scoliosis. J Thromb Haemost 2005;3:1094-5.

27. Stanitski CL, Whittlesey G, Thompson I, Stanitski DF, Mohan A. Clotting parameters in patients with adolescent idiopathic scoliosis undergoing posterior spinal fusion and instrumentation. J Pediatr Orthop B 1998;7:132-4. 